\title{
Health-related quality of life in peripheral blood stem cell donors and bone marrow donors: a prospective study in Japan
}

Ayumi Fujimoto, Ritsuro Suzuki, Katsumi Orihara, Minako Iida, Takuya Yamashita, Koji Nagafuji, Heiwa Kanamori, Yoshihisa Kodera, Koichi Miyamura, Shinichiro Okamoto \& Masayuki Hino

\begin{tabular}{|c|l|}
\hline Citation & International Journal of Hematology. 111(6); 840-850 \\
\hline Issue Date & $2020-06$ \\
\hline Published & $2020-03-14$ \\
\hline Type & Journal Article \\
\hline Textversion & Author \\
\hline Rights & $\begin{array}{l}\text { C The Japanese Society of Hematology. This is a post-peer-review, pre-copyedit } \\
\text { version of an article published in International Journal of Hematology. The original } \\
\text { publication is available at https://doi.org/10.1007/s12185-020-02852-7. } \\
\text { See The Japanese Society of Hematology Copyright Information. } \\
\text { http://www.jshem.or.jp/modules/en/index.php?content_id=14. } \\
\text { C 日本血液学会. この原稿は「私的使用」にかぎり利用できます。その他の利用には、 } \\
\text { 著作権者の許可が必要です。 http://www.jshem.or.jp/. }\end{array}$ \\
\hline 10.1007/s12185-020-02852-7 \\
\hline DOI
\end{tabular}

\section{Self-Archiving by Author(s)}

Placed on: Osaka City University Repository

Fujimoto, A., Suzuki, R., Orihara, K. et al. Health-related quality of life in peripheral blood stem cell donors and bone marrow donors: a prospective study in Japan. International Journal of Hematology. 111, 840-850 (2020). doi:10.1007/s12185-020-02852-7 
Original article

\section{Health-related Quality of Life in Peripheral Blood Stem Cell Donors and Bone Marrow Donors: A Prospective Study in Japan}

Running title: Health-related Quality of Life of Stem Cell Donors

Ayumi Fujimoto, ${ }^{1}$ Ritsuro Suzuki ${ }^{1}$, Katsumi Orihara ${ }^{2}$, Minako Iida ${ }^{3}$, Takuya Yamashita ${ }^{4}$, Koji Nagafuji ${ }^{5}$, Heiwa Kanamori ${ }^{6}$, Yoshihisa Kodera ${ }^{3}$, Koichi Miyamura ${ }^{7}$, Shinichiro Okamoto $^{8}$, and Masayuki Hino 9

${ }^{1}$ Department of Oncology and Hematology, Shimane University Hospital, Izumo, Japan; ${ }^{2}$ Japan Marrow Donor Program, Tokyo, Japan;

${ }^{3}$ Department of Promotion for Blood and Marrow Transplantation, Aichi Medical University School of Medicine, Nagakute, Japan;

${ }^{4}$ Department of Hematology, St. Luke’s International Hospital, Tokyo, Japan;

${ }^{5}$ Division of Hematology and Oncology, Department of Medicine, Kurume University School of Medicine, Kurume, Japan;

${ }^{6}$ Department of Hematology, Kanagawa Cancer Center, Yokohama, Japan;

${ }^{7}$ Department of Hematology, Japanese Red Cross Nagoya First Hospital, Nagoya, Japan;

${ }^{8}$ Division of Hematology, Department of Medicine, Keio University School of Medicine, Tokyo, Japan;

${ }^{9}$ Department of Hematology, Graduate School of Medicine, Osaka City University, Osaka, Japan.

Corresponding author: Masayuki Hino 
Department of Hematology, Graduate School of Medicine, Osaka City University, Osaka, Japan.

E-mail:hinom@med.osaka-cu.ac.jp

Phone: +81-6-6645-3881, Fax: +81-6-6645-3880

Word counts: text, 3,641; abstract, 199. Number of figures, 4; tables, 4; references, 16.

Keywords: health-related quality of life, stem cell donors, adverse events, risk factors 


\begin{abstract}
Understanding of the impact of stem cell donation on donors' health-related quality of life (HRQOL) remains limited. A prospective observational study of eligible unrelated donors enrolled in the Japan Marrow Donor Program was conducted to compare HRQOL and adverse events (AEs) between peripheral blood stem cell (PBSC) and bone marrow (BM) donors. In total, 107 PBSC donors and 108 BM donors were enrolled. HRQOL scores for physical status were significantly lower in BM donors one week post-harvest $(\mathrm{P}<0.001)$, but there were no significant differences between the two groups at baseline or three months post-harvest. PBSC donors were more likely to experience AEs before harvest $(\mathrm{P}<0.001)$. However, at harvest, moderate-to-severe AEs were more common in $\mathrm{BM}$ donors $(\mathrm{P}=0.001)$. After harvest, all grades of AEs were significantly higher in BM donors $(\mathrm{P}<0.001)$. Among $\mathrm{BM}$ donors, a lower total physical score at baseline (odds ratio (OR): 1.21), female sex (OR: 2.71), and young donors (OR: 3.08) were risk factors for moderate-to-severe AEs at harvest, while among PBSC donors, only female sex (OR: 4.86) was a risk factor. Our findings show better HRQOL during PBSC donation. These data help support decision-making by potential donors.
\end{abstract}




\section{Introduction}

Allogeneic hematopoietic stem cell transplantation is a curative treatment for many patients with hematological diseases. Human leukocyte antigen (HLA) allelic differences between recipients and donors induce allo-recognition by each $\mathrm{T}$ lymphocyte and result in graft-versus-host disease. Therefore, HLA genotypically matched sibling donors are preferred. However, only $\sim 30 \%$ of patients have such a donor and $70 \%$ of patients require an alternative donor.

In Japan, the Japan Marrow Donor Program (JMDP), which was established in 1991 [1], coordinates unrelated donors. The JMDP has continued to facilitate the coordination of unrelated donors [2]; the current donor pool is 509263 (as of 30 March 2019) and 23002 unrelated transplants have been performed to date. The JMDP began recruiting unrelated PBSC donors in October 2010. Recently, the numbers of PBSC unrelated donors have increased in many countries, including Japan [3]. However, the number of unrelated PBSC transplants undertaken remains low in Japan, with only 163 unrelated PBSC transplants performed in 2017, versus over 1000 unrelated BM transplants. We ascribe the small number of unrelated PBSC transplants to the delayed facilitation of granulocyte-colony stimulating factor (G-CSF) mobilized PBSC harvest from unrelated donors, following confirmation of the long-term safety of the harvest procedure for related donors [4].

Although both PBSC and BM donation are generally considered safe, donors often experience adverse events (AEs) such as pain and malaise[5], which can affect their health-related quality of life (HRQOL). Prospective studies of HRQOL associated with stem cell donation were recently reported $[6,7]$. However, detailed HRQOL and AE information comparing PBSC and BM donors remains limited. To further evaluate HRQOL and AEs, a prospective study was conducted using data from unrelated PBSC and BM donors enrolled in the JMDP. This study also aimed to provide evidence that 
may support decision-making among potential donors.

\section{Methods}

Study design and data source

This prospective observational study collected data from unrelated donors registered in the JMDP. This study was performed in accordance with the Declaration of Helsinki and approved by the JMDP Ethical Committee.

\section{Data collection}

Unrelated potential donors registered in the JMDP were contacted to undergo medical examination before donation. PBSCs or BM was donated according to the donor's preference, patient's condition, and attending physician's intention. Participants were consecutively recruited after confirming the donor's eligibility between January 2012 and September 2017.

All donors were required to be aged 20-54 years, with a body weight of at least 45 $\mathrm{kg}$ for men or $40 \mathrm{~kg}$ for women and a body mass index (BMI) $<30 \mathrm{~kg} / \mathrm{m}^{2}$. They were also required to have no medical history that might affect stem cell collection, including cancer, autoimmune diseases, organ dysfunction and hypertension. All donors underwent rigorous physical eligibility screening and provided informed consent for donation in accordance with normal practice, as well as additional informed consent for the self-report questionnaires to assess HRQOL and AEs at each point. Donors were considered eligible in this study if they were (1) satisfied with the criteria of physical screening, (2) first time donors at an authorized institution for BM or PBSC harvest, and (3) agreed to participate in this study before G-CSF injection for PBSC donors or before autologous blood collection for BM donors. A consent to participate was obtained from each donor in advance. 


\section{Stem cell collection}

All PBSC donors were hospitalized and received G-CSF based on the JMDP rule, including filgrastim $400 \mu \mathrm{g} / \mathrm{m}^{2}$ or lenograstim $10 \mu \mathrm{g} / \mathrm{kg}$, for 4 or 5 consecutive days, and apheresis was performed from day 4 or 5 . A maximum of two consecutive days' apheresis was permitted. All BM donors underwent harvest from both iliac crests of the pelvis under general anesthesia. The upper limit of the harvested BM volume was 15 $\mathrm{ml} / \mathrm{kg}$ of the patient's weight and did not exceed $20 \mathrm{ml} / \mathrm{kg}$ of the donor's weight.

\section{Questionnaires}

HRQOL was assessed using the 36-item short-form health survey (SF-36) questionnaire. This is the most commonly used HRQOL tool worldwide [8,9], and consists of eight categories, including four categories relating to physical status (physical function $[\mathrm{PF}]$, role functioning physical $[\mathrm{RP}]$, bodily pain $[\mathrm{BP}]$, and general health perception $[\mathrm{GH}]$ ) and four categories relating to mental status (vitality [VT], social functioning [SF], role functioning emotional [RE], and mental health $[\mathrm{MH}])$. The score for each category ranges from $0-100$, with lower scores indicating poorer health or greater disability. Donors completed the SF-36 questionnaire at three points: 1) baseline, i.e., before G-CSF injection for PBSC donors and before autologous blood collection for BM donors; 2) 1 week post-harvest; and 3$) 3$ months post-harvest.

AEs were assessed using self-reported questionnaires, which were provided to donors at three points: 1) pre-harvest, i.e., between G-CSF administration and donation for PBSC donors and between autologous blood collection and donation for BM donors; 2) the day of harvest; and 3) post-harvest, i.e., between donation and hospital discharge. AEs were graded as severe, moderate, mild, slight, and none based on the Common Terminology Criteria for Adverse Events version 4.0. 
After confirming the donor's eligibility, the first HRQOL questionnaire was sent directly by JMDP to eligible donors at the predefined time-point and then sent back by consented donors. Results were tallied up by the JMDP staff. The first questionnaire was sent to PBSC donors before G-CSF administration and to BM donors before autologous blood collection. Since the time between donor health checkup and sending the questionnaire was shorter for BM donors than for PBSC donors, the first questionnaire could not be sent to some BM donors. Therefore, the number of the first questionnaires distributed during the study period was almost the same for both PBSC and BM donors with each 300 subjects. The rate of response was approximately $40 \%$, and was equivalent by donor types. Subsequent questionnaires were sent to donors who answered the first one. AE questionnaires were handed over to each donor by a donor coordinator, not through the primary physician.

\section{Statistical analysis}

The primary endpoint was to compare HRQOL between unrelated PBSC and BM donors. The secondary endpoints were to compare AEs and hospitalization periods between the two groups, and to identify risk factors for the development of AEs. Differences in HRQOL were assessed using repeated measures analysis of variance and covariance. Differences in HRQOL values in each category at different time points were examined using the Mann-Whitney U test. AEs were compared between the two groups using the $\chi^{2}$ test. To identify risk factors, logistic regression analysis was performed, and odds ratios (ORs) with 95\% confidential interval (CI) were calculated for each variable. Pre-harvest total physical score was treated as a continuous variable. The OR of pre-harvest total physical score was calculated for a 10 score decrease. As described previously [6], pre-harvest total physical score was also categorized into quartiles when calculating its OR. The hospitalization period was compared using the Mann-Whitney U 
test. A two-sided $P$-value $<0.05$ was considered statistically significant. All statistical analyses were performed using STATA version 14.0 (StataCorp., College Station, TX, USA). 


\section{Results}

Characteristics of donors

Answers to the questionnaires were received from 215 donors, including 107 PBSC donors and 108 BM donors. Their characteristics and donors who did not participate this study but donated stem cells during the same period are shown in Table 1. The median age of PBSC and BM donors included in the current study was 37 years (range, 21-53 years) and 39 years (range, 21-54 years), respectively. The percentage of males was higher in the PBSC group (73\%) than in the BM group $(53 \% ; P=0.02)$. The year of donation was equally distributed in BM donors, but was slanted toward recent years in PBSC donors $(P<0.001)$. Other baseline variables did not significantly differ between the two groups. Only $2(1.9 \%)$ of 107 PBSC donors underwent femoral vein cannulation for apheresis.

\section{Hospitalization duration}

The median hospitalization duration was 6 days (range, 3-8 days) in PBSC donors, but 4 days (range, 3-6 days) in BM donors. The hospitalization duration was significantly longer in PBSC donors than in BM donors $(P<0.001$; Figure 1$)$.

\section{$H R Q O L$}

All participants completed the SF-36 questionnaire on at least one of the three time points. The return rates of the completed questionnaires from donors who answered the first questionnaire are shown in Table 2. The scores of each SF-36 category at different time points showed no significant differences between PBSC and BM donors at baseline (Figure S1). However, at 1 week post-harvest, the physical status scores, including PF, $\mathrm{RP}$, and BP, were significantly lower in BM donors than in PBSC donors $(P<0.001$; Table 3). SF, a mental status score, was also significantly lower in BM donors than in 
PBSC donors $(P=0.03)$. At 3 months post-harvest, the scores recovered and did not significantly differ between the two groups.

The longitudinal SF-36 scores in each category are shown in Figure 2. Among BM donors, the PF, RP, and BP physical status scores and SF and RE mental status scores were significantly lower at 1 week post-harvest than at baseline. Among PBSC donors, however, only RP and BP physical status scores were significantly reduced, and no categories of mental status were changed from baseline. Among the eight categories of the SF-36 questionnaire, only MH increased at 1 week post-harvest in both groups.

AEs

Major AEs reported in each group are shown in Figure 4. Before harvest, the most common $\mathrm{AE}$ was pain at the donation site $(23 \%)$ in $\mathrm{BM}$ donors, and low back pain (75\%) due to G-SCF administration in PBSC donors. The incidence of all-grade and moderate-to-severe grade AEs before harvest was significantly higher in PBSC donors than in BM donors $(P<0.001$ and $P<0.001$, respectively). On the day of harvest, BM donors most commonly reported pain at the donation site (86\%), low back pain (71\%), and throat pain (73\%). By contrast, PBSC donors most commonly reported low back pain $(82 \%)$ and general malaise $(64 \%)$. The percentage of reported all-grade AEs at harvest was not significantly different between PBSC and BM donors $(P=0.30)$. However, the rate of moderate-to-severe AEs was significantly higher in BM donors than in PBSC donors $(P=0.001)$. After harvest, low back pain and pain at the donation site were common AEs in both groups. The probability of all-grade and moderate-to-severe AEs after harvest was significantly higher in BM donors than in PBSC donors $(P<0.001$ and $P=0.002$, respectively; Table 4). After discharge, medical follow-up was offered to all donors within a few weeks. Among 107 PBSC donors, seven $(6.5 \%)$ were followed up more than once, due to abnormal laboratory test results 
$(\mathrm{N}=4)$, pain in the right upper quadrant $(\mathrm{N}=1)$, cough and throat pain $(\mathrm{N}=1)$, and general malaise $(\mathrm{N}=1)$. Among $108 \mathrm{BM}$ donors, three $(2.8 \%)$ were followed up more than once because of pain at the donation site $(\mathrm{N}=2)$ and abnormal laboratory test results $(\mathrm{N}=1)$. With the exception of a PBSC donor who experienced persistent general malaise, follow-up was discontinued at the second assessment as symptoms had resolved.

\section{Factors affecting AEs}

$\mathrm{BM}$ donors with lower total physical scores (PF, RP, BP, and $\mathrm{GH}$ ) at baseline experienced significantly more moderate-to-severe AEs at harvest (OR 1.21 for a 10 score decrease, 95\% CI 1.03-1.42, $P=0.02$ ). Among these physical categories, lower $\mathrm{PF}$ and lower BP at baseline were significantly associated with the development of moderate-to-severe AEs at harvest (OR 3.64 for a 10 score decrease, 95\% CI 1.10-12.1, $P=0.04$, and OR 1.34 for a 10 score decrease, 95\% CI 1.04-1.72, $P=0.02$, respectively). No mental category was associated with the development of moderate-to-severe AEs at harvest. Regarding the other variables, female donors or young donors aged $\leq 30$ years were more likely to experience moderate-to-severe AEs at harvest (OR 3.11, 95\% CI 1.38-6.97, $P=0.006$ and OR 3.25, 95\% CI 1.22-8.68, $P=$ 0.02, respectively). Logistic regression analysis showed that a lower total physical score of HRQOL at baseline (OR 1.21 for a 10 score decrease, 95\% CI 1.02-1.43, $P=0.03$ ), female sex $(\mathrm{OR} 2.71,95 \% \mathrm{CI} 1.15-6.36, P=0.02)$ and young donors $(\mathrm{OR} 3.08,95 \% \mathrm{CI}$ 1.07-8.84, $P=0.04)$ were risk factors for the development of moderate-to-severe AEs at harvest. However, none of these factors were associated with moderate-to-severe AEs after harvest. The impact of a pre-harvest total physical score remained significant, even when compared with the lowest quartile of the pre-harvest total physical score and the other quartiles (OR 2.93, 95\% CI 1.07-8.04, $P=0.04)$. 
Among PBSC donors, no mental or physical category was associated with the development of moderate-to-severe AEs at harvest. Only female sex was identified as a risk factor for moderate-to-severe AEs at harvest (OR 4.86, 95\% CI 1.89-12.5, $P=$ $0.001)$, but this association did not persist after harvest.

Among all donors, logistic regression analysis revealed that BM donor (OR 2.40, 95\% CI 1.26-4.57, $P=0.008)$ was identified as an independent risk factor for the development of AEs at harvest, as well as other variables, including female sex (OR $3.44,95 \%$ CI $1.81-6.54, P<0.001)$, younger age (OR 2.25, 95\% CI 1.08-4.67, $P=$ 0.03), and a lower total physical score of HRQOL at baseline (OR 1.11 for a 10 score decrease, 95\% CI 1.006-1.22, $P=0.04)$. In addition, the development of moderate-to-severe AEs at harvest significantly decreased the total physical score and total mental score 1 week after harvest in both BM and PBSC donors (Figure 4). However, the impacts lasted until 3 months after harvest only for BM donors, but not in PBSC donors. 


\section{Discussion}

As the number of allogeneic hematopoietic stem cell transplantations from unrelated donors increases $[10,11]$, the need to facilitate stem cell donation also increases. Voluntary donation of BM or PBSCs is a well-established altruistic act and the safety of these processes has been widely evaluated. However, even if AEs are not severe, donors may experience affliction, anxiety and disability, that can impact their HRQOL. Therefore, it is important to evaluate not only AEs but also HRQOL of donors, as information on this aspect of the donor experience remains limited.

This prospective study examined differences in HRQOL and AEs between PBSC and BM donors. No significant differences were seen at baseline in HRQOL parameters, but BM donors had a slight advantage over PBSC donors in that they did not experience low back pain due to G-CSF administration before harvest. However, the percentage of donors with moderate-to-severe AEs, particularly pain at the donation site, was significantly higher among BM donors on the day of harvest and this pain continued until discharge. Subsequently, at 1 week post-harvest, HRQOL related to physical status was significantly lower in BM donors than in PBSC donors. However, HRQOL values had recovered by 3 months post-harvest and did not differ between PBSC and BM donors at this point.

Due to the more severe AEs among BM donors, physical HRQOL significantly declined just after harvest, which is consistent with previous studies of HRQOL in BM donors $[6,7,12,13]$. The SF score also decreased significantly in BM donors versus PBSC donors. This may have resulted from pain at the donation site and low back pain due to the harvest procedure in BM donors. This seriously affected donors' daily activities, and might lead to difficulty in working normally. A recent prospective study reported that, besides the SF-36 categories discussed above, mental HRQOL, including VT and RE, was significantly lower in BM donors than in PBSC donors [6]. VT and RE 
did not differ between the two procedures in our study. The different results might be affected by the differences of donor characteristics and the duration of hospitalization in PBSC donors between the previous study and the current study. A previous study showed that donors who experienced physical difficulties with donation felt less psychologically positive about donation. Therefore, the more severe physical AEs in BM donors may explain the decrease in their mental status[14]. A recently reported phase III randomized trial of unrelated stem cell donors showed that recovery time and long-term HRQOL consequences did not differ between the two donation procedures[7]. Similar findings were reported in another study[15]. By contrast, other prospective studies showed that, due to the more severe AEs experienced at donation, the recovery time of BM donors was significantly longer than that of PBSC donors[6, 13, 16]. In comparison with these previous studies, HRQOL in the two donor groups evaluated in our study had recovered at an earlier stage (by 3 months post-harvest). Therefore, no delay in the recovery of BM donors was evident, although an accurate recovery time was not evaluated. In the phase III trial, the HRQOL score was not different between $\mathrm{BM}$ and PBSC donors, although the physical score of BM donors was lower than that of PBSC donors at 48 hours post-harvest. These results were not consistent with our findings, which showed a significantly lower physical score for BM donors at harvest. This might be derived from the difference of time of HRQOL evaluation around harvest between the two studies. HRQOL was evaluated on the same day as harvest in our study, which allowed the effect of each harvest procedure on each donor's HRQOL to be evaluated in more accurate.

During the donation period, almost all donors experienced at least one AE. Before harvest, the incidence of AEs was significantly higher in PBSC donors than in BM donors. The major AEs in the PBSC group included low back pain and general malaise, and these moderate-to-severe grade AEs occurred in $26 \%$ and $12 \%$ of PBSC donors, 
respectively. These frequencies are higher than those in related PBSC donors reported from the Japan Society for Hematopoietic Cell Transplantation (16\% and 4.5\%, respectively) [4], but similar to those in unrelated PBSC donors reported from the National Marrow Donor Program [5]. The incidence of AEs was significantly higher in BM donors than in PBSC donors on the day of harvest, particularly pain at the donation site and low back pain. More than $80 \%$ of BM donors experienced pain at the donation site, $30 \%$ of which were moderate-to-severe AEs. These results are also consistent with the previous findings [5]. On discharge from hospital, 33\% of BM donors still had moderate-to-severe AEs, whereas $14 \%$ of PBSC donors had these AEs, i.e., the symptoms lasted longer in BM donors. After discharge, only one PBSC donor with persistent general malaise underwent medical examinations more than twice, and thus AEs could be considered transient in most donors. Stem cell donation is accompanied by the risk of rare but life-threatening AEs [17], but no critical AEs were seen in our study.

Regarding moderate-to-severe AEs at harvest, a lower total physical score at baseline, female sex, and young donors were identified as risk factors among BM donors. These results are consistent with previous reports that donors with pre-donation SF-36 physical component summary scores in the lowest quartile were more likely to experience fatigue or any AEs than those with scores in the highest quartile [6]. Another study showed that female sex is associated with lower PF, RP, and BP at harvest [12]. Female donors were more likely to experience AEs at harvest, which may have affected these results, although an association between female sex and lower physical HRQOL was not observed in our study (data not shown). By contrast, only female sex was identified as a risk factor among PBSC donors, and physical status was not associated with AE development. A prospective study of related PBSC donors reported that female sex was the only risk factor for fatigue, headache, insomnia, anorexia, or nausea [4], 
which is generally consistent with our results. Female sex was identified as a risk factor for AEs at harvest in both groups, indicating that female donors are more sensitive to pain regardless of the donation procedure. Therefore, it is advisable to focus on careful management of AEs, particularly in donors with these risk factors. Regarding the association between pre-donation mental status and AEs at harvest, pre-donation total mental scores in the lowest quartile were reported to be associated with a higher level of pain during donation than those in the highest quartile [6]. However, in our study, mental status at baseline was not related with AE development in either group.

The hospitalization duration was significantly longer in PBSC donors than in BM donors. This is because PBSC donors are usually hospitalized on day 1 of G-CSF administration while BM donors are typically hospitalized 1 day before donation in Japan. The inconvenience of longer hospitalization for PBSC donors could be partially alleviated by administrating G-CSF in an outpatient department.

The questionnaire return rates were acceptable at over $80 \%$ during all tested points. The number of donors was similar in each group, enabling appropriate comparison of HRQOL and AEs at all three time points. However, there are several limitations. First, the SF-36 score reflects only part of overall HRQOL. Second, the number of donors was limited ( $\sim 110$ per group). Therefore, to evaluate HRQOL of donors in more detail, a larger study using more comprehensive HRQOL assessment tools is required. Third, the year of harvest was not balanced between each donor due to the historical delay of the introduction of PBSC harvest in Japan. In addition, there were significant differences in sex and the year of harvest between donors who participated in the current study and those who did not in the same period.

In conclusion, this prospective study of unrelated stem cell donors clearly demonstrated differences between PBSC and BM donors and longitudinal changes in HRQOL and AEs during donation. Our results will be useful for the recruitment of 
potential donors and in explaining how donation can result in AEs and poor HRQL. Our study will also be useful in helping donors chose between PBSC donation and BM donation. We believe that the results of the present study will facilitate decision-making among potential donors and stem cell donation in the future. 


\section{Conflict of interest}

A.F. received honoraria from Bristol-Meyer Squib and Celgene. R.S. received honoraria from Kyowa Hakko Kirin and Chugai Pharmaceutical. The other authors declare no competing financial interests. M.I. Dr. Iida received donation to the endowed chair from Kyowa Kirin Co., Ltd., JCR Pharmaceuticals Co., Ltd., Clinigen K.K., Astellas Pharma Inc., Genzyme Japan K.K., CHUGAI PHARMACEUTICAL CO., LTD., Janssen Pharmaceutical K.K., Sanofi K.K., Otsuka Pharmaceutical Co., Ltd. K.N. received grants from Kyowa Kirin and from Chugai Pharma. K.M. received honoraria from Bristol Myers, Novartis, Pfizer, Astellas, Takeda, Otsuka, Celgene, Dainippon-Sumitomo, Kyowa-Hakko Kirin, Chugai, MSD, and Alexon. S.O. received grants from Asahi Kasei Pharma, Astellas, Eisai, Otsuka, Ono, Kyowa Kirin, Sanofi, Shionogi, Daiichi Sankyo, Takeda, Dainihon Sumitomo, Chugai, Teijin Pharma, Novartis, Mochida, JCR Pharma, and Pfizer, and received honoraria from Astellas, Eisai, Otsuka, Ono, Kyowa Kirin, Sanofi, Daiichi Sankyo, Takeda, Chugai, Nihon Shinyaku, Novartis, BMS, Mochida, JCR Pharma, and Pfizer. M.H. received grants from Kyowa-Kirin, Chugai, Otsuka, Astellas, Takeda, Taiho, Teijin, MSD, Sumitomo Dainippon, Pfizer, Eisai, Japan Blood Products Organization, and Nihon Pharmaceutical, and received honoraria from Kyowa-Kirin, Chugai, Otsuka, Astellas, Takeda, MSD, Sumitomo Dainippon, Pfizer, Bristol-Myers Squibb, Daiichisankyo, Jansenn, Celgene, Mochida, Ono, Sanofi, Nippon Shinyaku, Mundi Pharma, and Alexion. The other authors declare no competing financial interests.

\section{Acknowledgements}

We thank all the physicians who helped to gather data from donors and data managers at the JMDP. This work was supported in part by the Practical Research 
Project for Allergic Disease and Immunology (Research Technology of Medical Transplantation) from the Ministry of Health, Labor and Welfare of Japan.

Author contributions: R.S., M.I., T.Y., K.N., H.K., Y.K., K.M. and M.H. designed the study and interpreted data. K.O., M.H. performed the research and data collection. A.F. and R.S. performed analyses and interpreted data. A.F. wrote the manuscript and R.S., Y.K., S.O. and M.H. revised the manuscript. All authors discussed the results and reviewed the manuscript and approved the final version. 


\section{References}

1. Kodera Y. The Japan Marrow Donor Program, the Japan Cord Blood Bank Network and the Asia Blood and Marrow Transplant Registry. Bone Marrow Transplant 2008; 42 Suppl 1: S6.

2. Saito H, Ito M, Kato S, Kodera Y, Okamoto S, Taniguchi S et al. The Japan Marrow Donor Program, 25 years of experience in achieving 20,000 bone marrow transplantations: organization structure, activity, and financial basis. Bone Marrow Transplant 2018; 53: 609-616.

3. Goto T, Tanaka T, Sawa M, Ueda Y, Ago H, Chiba S et al. Prospective observational study on the first 51 cases of peripheral blood stem cell transplantation from unrelated donors in Japan. Int J Hematol 2018; 107: 211-221.

4. Kodera Y, Yamamoto K, Harada M, Morishima Y, Dohy H, Asano S et al. PBSC collection from family donors in Japan: a prospective survey. Bone Marrow Transplant 2014; 49: $195-200$.

5. Pulsipher MA, Chitphakdithai P, Logan BR, Shaw BE, Wingard JR, Lazarus HM et al. Acute toxicities of unrelated bone marrow versus peripheral blood stem cell donation: results of a prospective trial from the National Marrow Donor Program. Blood 2013; 121: 197-206.

6. Billen A, Madrigal JA, Strydom A, Szydlo RM, Switzer GE, Shaw BE. Predonation health-related quality of life scores predict time to recovery in hematopoietic stem cell donors. Biol Blood Marrow Transplant 2015; 21: 350-356.

7. Switzer GE, Bruce JG, Harrington D, Haagenson M, Drexler R, Foley A et al. Health-related quality of life of bone marrow versus peripheral blood stem cell donors: a prespecified subgroup analysis from a phase III RCT-BMTCTN protocol 0201. Biol Blood Marrow Transplant 2014; 20: 118-127.

8. Garratt A, Schmidt L, Mackintosh A, Fitzpatrick R. Quality of life measurement: 
bibliographic study of patient assessed health outcome measures. Bmj 2002; 324: 1417.

9. Ware JE, Jr., Sherbourne CD. The MOS 36-item short-form health survey (SF-36).

I. Conceptual framework and item selection. Med Care 1992; 30: 473-483.

10. Aljurf M, Weisdorf D, Alfraih F, Szer J, Muller C, Confer D et al. "Worldwide Network for Blood \& Marrow Transplantation (WBMT) special article, challenges facing emerging alternate donor registries". Bone Marrow Transplant 2019; 54: 1179-1188.

11. Niederwieser D, Baldomero H, Szer J, Gratwohl M, Aljurf M, Atsuta Y et al. Hematopoietic stem cell transplantation activity worldwide in 2012 and a SWOT analysis of the Worldwide Network for Blood and Marrow Transplantation Group including the global survey. Bone Marrow Transplant 2016; 51: 778-785.

12. Nishimori M, Yamada Y, Hoshi K, Akiyama Y, Hoshi Y, Morishima Y et al. Health-related quality of life of unrelated bone marrow donors in Japan. Blood 2002; 99: 1995-2001.

13. Rowley SD, Donaldson G, Lilleby K, Bensinger WI, Appelbaum FR. Experiences of donors enrolled in a randomized study of allogeneic bone marrow or peripheral blood stem cell transplantation. Blood 2001; 97: 2541-2548.

14. Switzer GE, Simmons RG, Dew MA. Helping Unrelated Strangers: Physical and Psychological Reactions to the Bone Marrow Donation Process Among Anonymous Donors1. Journal of Applied Social Psychology 1996; 26: 469-490.

15. Kennedy GA, Morton J, Western R, Butler J, Daly J, Durrant S. Impact of stem cell donation modality on normal donor quality of life: a prospective randomized study. Bone Marrow Transplant 2003; 31: 1033-1035.

16. Bredeson C, Leger C, Couban S, Simpson D, Huebsch L, Walker I et al. An evaluation of the donor experience in the canadian multicenter randomized trial of bone marrow versus peripheral blood allografting. Biol Blood Marrow Transplant 2004; 10: 405-414. 
17. Halter J, Kodera Y, Ispizua AU, Greinix HT, Schmitz N, Favre G et al. Severe events in donors after allogeneic hematopoietic stem cell donation. Haematologica 2009; 94: $94-101$. 
Table 1 Demographic details of donors

\begin{tabular}{|c|c|c|c|c|c|c|c|c|c|c|}
\hline & \multicolumn{4}{|c|}{ Registered donors $(\mathrm{N}=215)$} & \multirow[b]{2}{*}{$\begin{array}{c}P(\text { PBSC vs } \\
\text { BM })\end{array}$} & \multicolumn{4}{|c|}{ Non-registered unrelated donors $(\mathrm{N}=7,261)$} & \multirow[b]{2}{*}{$\begin{array}{l}P(\text { registered } \mathrm{v} \\
\text { non-registered) }\end{array}$} \\
\hline & PBSC & $(\%)$ & BM & $(\%)$ & & PBSC & $(\%)$ & BM & $(\%)$ & \\
\hline Number & 107 & & 108 & & & 267 & & 6,994 & & \\
\hline \multicolumn{11}{|l|}{ Sex } \\
\hline Male & 78 & (73) & 57 & (53) & 0.02 & 205 & (77) & 4,867 & (70) & 0.03 \\
\hline Female & 29 & (27) & 51 & (47) & & 62 & (23) & 2,127 & (30) & \\
\hline \multicolumn{11}{|c|}{ Donor age at donation, yrs } \\
\hline $20-29$ & 24 & (22) & 23 & (21) & & 30 & (11) & 1,196 & (17) & \\
\hline $30-39$ & 41 & (38) & 35 & (33) & & 95 & (36) & 2,527 & (36) & \\
\hline $40-49$ & 35 & (33) & 40 & (37) & & 115 & (43) & 2,647 & (38) & \\
\hline $50-55$ & 7 & (7) & 10 & (9) & & 27 & (10) & 624 & (9) & \\
\hline Median (range) & 37 & $(21-53)$ & 39 & $(21-54)$ & 0.21 & 40 & $(20-55)$ & 39 & $(20-55)$ & 0.12 \\
\hline \multicolumn{11}{|l|}{ Donor BMI, $\mathrm{kg} / \mathrm{m}^{2}$} \\
\hline Underweight, $<18.5$ & 3 & (3) & 6 & (5) & & 5 & (2) & 248 & (4) & \\
\hline Normal, 18.5-24.9 & 80 & (75) & 85 & (79) & & 209 & (78) & 5,231 & (75) & \\
\hline Overweight, $>25$ & 24 & (22) & 17 & (16) & & 53 & (20) & 1,515 & (22) & \\
\hline Median (range) & 22.2 & $(17.2-29.1)$ & 21.9 & $(15.8-29.0)$ & 0.43 & 22.7 & $(17.0-29.4)$ & 22.7 & $(15.4-30.4)$ & 0.004 \\
\hline \multicolumn{11}{|c|}{ Donor WBC counts, $\times 10^{3}$} \\
\hline Median (range) & 5.5 & $(3.5-9.4)$ & 5.5 & $(3.2-10.0)$ & 0.99 & 5.4 & $(2.7-11.2)$ & 5.4 & $(2.4-17.6)$ & 0.19 \\
\hline \multicolumn{11}{|l|}{ Donor hemoglobin level } \\
\hline $\begin{array}{l}\text { Median, male } \\
\text { (range) }\end{array}$ & 15.3 & $(13.1-17.8)$ & 15.2 & $(13.2-17.4)$ & 0.72 & 15 & $(12.6-17.7)$ & 14.1 & $(10.2-17.1)$ & $<0.001$ \\
\hline $\begin{array}{l}\text { Median, female } \\
\text { (range) }\end{array}$ & 13.3 & $(12.0-15.2)$ & 13.0 & $(12.0-15.3)$ & 0.29 & 13.1 & $(11.5-14.7)$ & 12.3 & $(8.8-16.0)$ & 0.73 \\
\hline \multicolumn{11}{|c|}{ Donor platelet counts, $\times 10^{4}$} \\
\hline Median (range) & 23.9 & $(16.5-35.5)$ & 22.9 & $(15.3-33.9)$ & 0.86 & 23.3 & $(11.6-41.6)$ & 25 & $(9.4-49.9)$ & 0.14 \\
\hline \multicolumn{11}{|l|}{ Year of donation } \\
\hline 2011-2012 & 10 & (9) & 49 & (45) & $<0.001$ & 4 & (2) & 1,269 & (18) & $<0.001$ \\
\hline 2013-2014 & 47 & (44) & 37 & (34) & & 25 & (9) & 2,578 & (37) & \\
\hline $2015-2017$ & 50 & (47) & 22 & (20) & & 238 & (89) & 3,147 & (45) & \\
\hline
\end{tabular}

BMI, body mass index; WBC, white blood cell; PBSC, peripheral blood stem cell; BM, bone marrow. 
Table 2 Type and timing of questionnaires and return rate

\begin{tabular}{|c|c|c|c|c|c|c|c|}
\hline \multirow{2}{*}{$\begin{array}{l}\text { Timing } \\
\text { Baseline }\end{array}$} & \multirow{2}{*}{$\begin{array}{c}\text { Type of } \\
\text { questionnaire } \\
\text { HRQOL }\end{array}$} & \multirow{2}{*}{$\begin{array}{c}\text { PBSC donors } \\
\text { Before G-CSF administration }\end{array}$} & \multicolumn{2}{|c|}{$\begin{array}{l}\text { Number of donors } \\
\text { (rate, \%) }\end{array}$} & $\mathrm{BM}$ donors & \multicolumn{2}{|c|}{$\begin{array}{l}\text { Number of donors } \\
\text { (rate, \%) }\end{array}$} \\
\hline & & & 107 & $(100)$ & $\begin{array}{l}\text { Prior to autologous blood } \\
\text { collection }\end{array}$ & 108 & $(100)$ \\
\hline Pre-harvest & AEs & $\begin{array}{l}\text { Between G-CSF } \\
\text { administration and harvest }\end{array}$ & 102 & $(95)$ & $\begin{array}{c}\text { Between autologous blood } \\
\text { collection and harvest }\end{array}$ & 101 & $(94)$ \\
\hline Harvest day & AEs & Just after harvest & 101 & $(94)$ & Just after harvest & 101 & $(94)$ \\
\hline Post-harvest & AEs & $\begin{array}{c}\text { Between harvest and hospital } \\
\text { discharge }\end{array}$ & 101 & $(94)$ & $\begin{array}{c}\text { Between harvest and hospital } \\
\text { discharge }\end{array}$ & 101 & $(94)$ \\
\hline & HRQOL & 1 week after harvest & 96 & $(90)$ & 1 week after harvest & 102 & $(94)$ \\
\hline & HRQOL & 3 months after harvest & 87 & $(81)$ & 3 months after harvest & 93 & $(86)$ \\
\hline
\end{tabular}

HRQOL, health-related quality of life; AE, adverse event; PBSC, peripheral blood stem cell; G-CSF, granulocyte-colony stimulating factor; BM, bone marrow. 
Table 3 SF-36 questionnaires in PBSC donors and BM donors

\begin{tabular}{|c|c|c|c|c|}
\hline Test point & $\begin{array}{l}\text { SF-36 } \\
\text { category }\end{array}$ & $\begin{array}{l}\text { PBSC } \\
\text { mean }\end{array}$ & BM mean & $P$ \\
\hline \multirow[t]{8}{*}{ Baseline } & $\mathrm{PF}$ & 97.9 & 97.7 & 0.91 \\
\hline & $\mathrm{RP}$ & 98.2 & 97.3 & 0.09 \\
\hline & $\mathrm{BP}$ & 86.6 & 86.7 & 0.64 \\
\hline & $\mathrm{GH}$ & 78.6 & 80.0 & 0.57 \\
\hline & VT & 68.7 & 69.6 & 0.83 \\
\hline & SF & 94.4 & 92.0 & 0.11 \\
\hline & $\mathrm{RE}$ & 93.5 & 94.8 & 0.54 \\
\hline & MH & 78.6 & 77.1 & 0.44 \\
\hline \multirow[t]{8}{*}{1 week post-harvest } & $\mathrm{PF}$ & 95.4 & 83.0 & $<0.001$ \\
\hline & $\mathrm{RP}$ & 86.7 & 73.8 & $<0.001$ \\
\hline & $\mathrm{BP}$ & 79.1 & 62.3 & $<0.001$ \\
\hline & $\mathrm{GH}$ & 78.4 & 75.6 & 0.15 \\
\hline & VT & 68.1 & 65.9 & 0.19 \\
\hline & $\mathrm{SF}$ & 87.9 & 83.5 & 0.03 \\
\hline & $\mathrm{RE}$ & 91.8 & 87.8 & 0.08 \\
\hline & MH & 81.5 & 80.7 & 0.97 \\
\hline \multirow[t]{8}{*}{3 months post-harvest } & $\mathrm{PF}$ & 99.0 & 98.2 & 0.31 \\
\hline & $\mathrm{RP}$ & 97.3 & 97.8 & 0.36 \\
\hline & $\mathrm{BP}$ & 92.5 & 90.3 & 0.33 \\
\hline & $\mathrm{GH}$ & 80.9 & 80.9 & 0.93 \\
\hline & VT & 71.7 & 72.4 & 0.84 \\
\hline & SF & 96.8 & 96.0 & 0.60 \\
\hline & $\mathrm{RE}$ & 96.1 & 96.4 & 0.94 \\
\hline & MH & 80.7 & 82.2 & 0.70 \\
\hline
\end{tabular}

SF-36, 36-item short-form health survey; PBSC, peripheral blood stem cells; BM, bone marrow; PF, physical function; RP, role functioning physical; BP, bodily pain; GH, general health perception; VT, vitality; SF, social functioning; RE, role functioning emotional; $\mathrm{MH}$, mental health. 
Table 4 Maximum grade of adverse events in each donor

\begin{tabular}{lcccccccc} 
Test point & Donor & $\begin{array}{c}\text { None } \\
(\%)\end{array}$ & $\begin{array}{c}\text { Slight } \\
(\%)\end{array}$ & $\begin{array}{c}\text { Mild } \\
(\%)\end{array}$ & $\begin{array}{c}\text { Moderate } \\
(\%)\end{array}$ & $\begin{array}{c}\text { Severe } \\
(\%)\end{array}$ & All grades & Moderate-to-severe \\
\hline Pre-harvest & PBSC & 17 & 19 & 36 & 25 & 3 & $<0.001$ & $<0.001$ \\
& BM & 70 & 23 & 5 & 2 & 0 & & 0.001 \\
Harvest day & PBSC & 10 & 21 & 40 & 22 & 7 & 0.30 & 0.002 \\
& BM & 6 & 16 & 27 & 34 & 17 & & \\
Post-harvest & PBSC & 20 & 39 & 27 & 9 & 5 & $<0.001$ & \\
& BM & 1 & 30 & 36 & 30 & 3 & & \\
\hline
\end{tabular}

PBSC, peripheral blood stem cells; BM, bone marrow. 


\section{Figure legends}

Figure 1 Comparison of hospitalization period in each donor group

The median duration of hospitalization in PBSC and BM donors was 6 and 4 days, respectively $(P<0.001)$. 


\section{Figure 1}

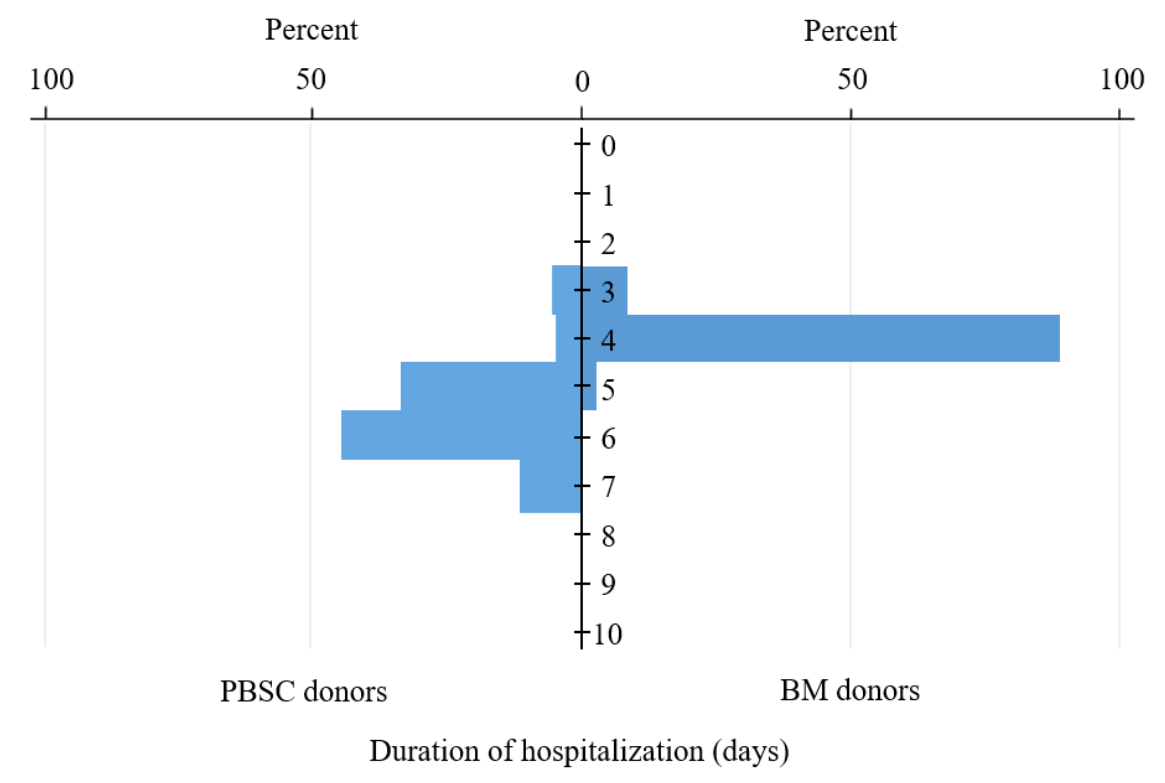

PBSC, peripheral blood stem cells; BM, bone marrow. 


\section{Figure 2 Longitudinal SF-36 score in each category}

The differences among the tested time points in each donor group and between PBSC and BM donors in each SF-36 category are shown. (A) Physical category and (B) mental category. PF, RP, and BP were significantly lower in BM donors than in PBSC donors (all $P<0.001$ ). Among BM donors, PF, RP, BP, SF, and RE were significantly lower at 1 week post-harvest than at baseline. Among PBSC donors, RP and BP were significantly lower at 1 week post-harvest than at baseline. 


\section{Figure 2}

\section{A. Physical status}

PF [Physical function]

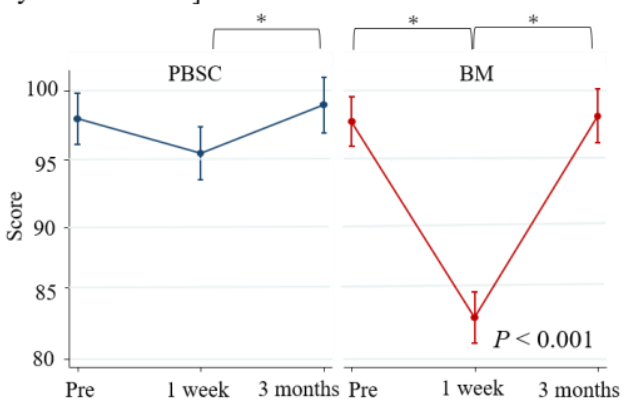

RP [Role functioning physical]

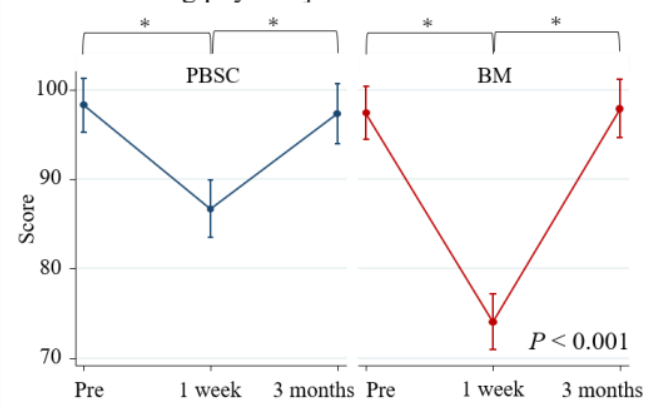

BP [Bodily pain]

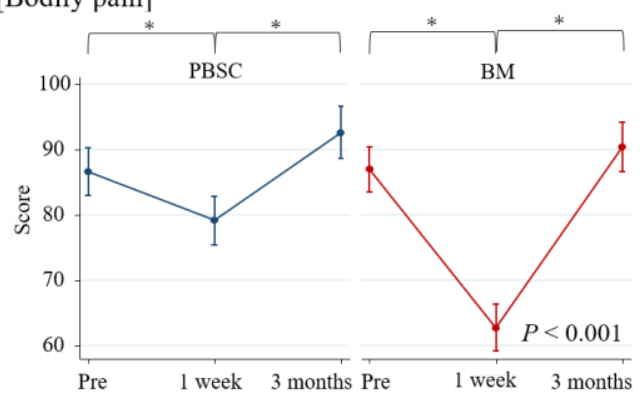

B. Mental status

VT [Vitality]

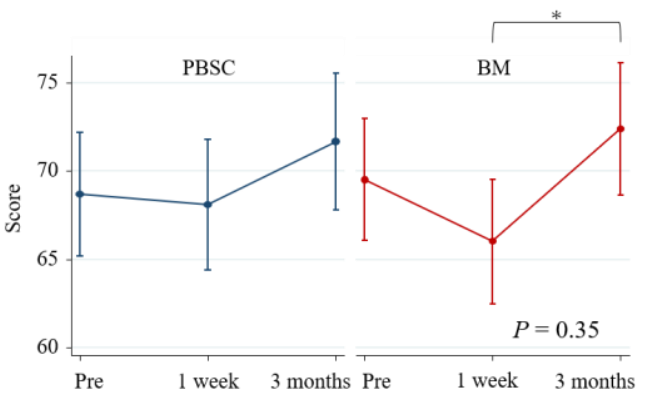

$\mathrm{RE}$ [Role functioning emotional]

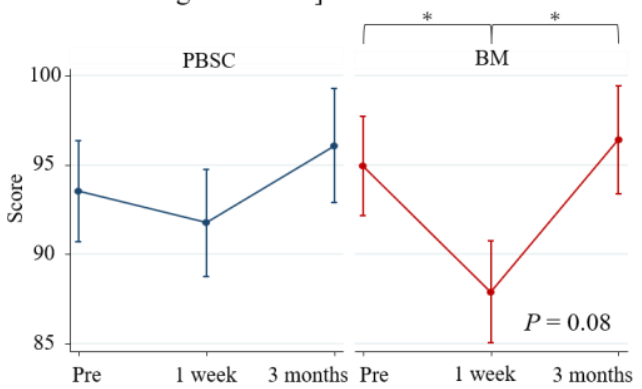

$* P<0.05$
GH [General health perception]

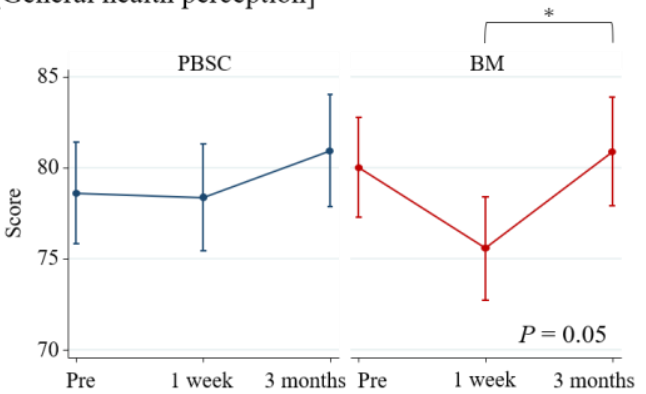

SF [Social functioning]

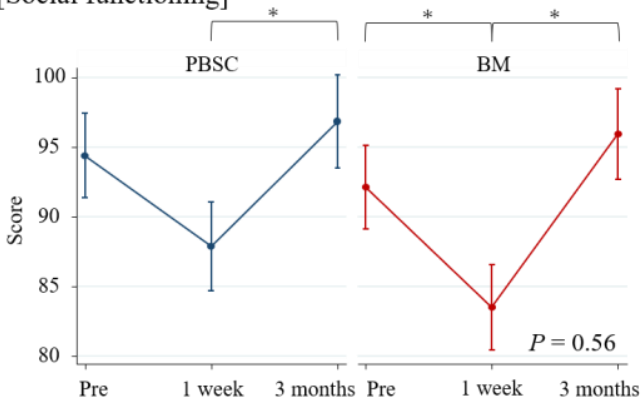

$\mathrm{MH}[$ Mental health]

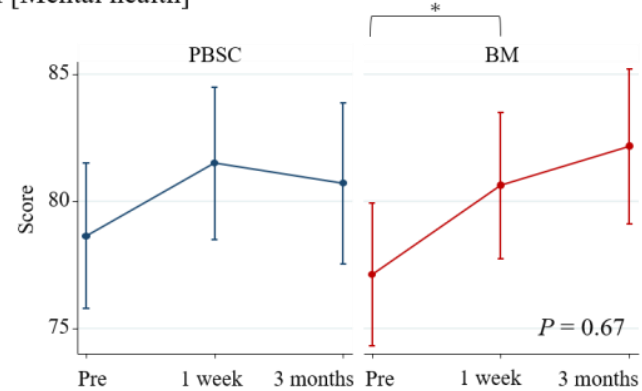

PBSC, peripheral blood stem cells; BM, bone marrow. 


\section{Figure 3 AEs at each test point}

The percentages of common AEs in each donor group are shown according to the grades. The darker the color, the more severe the AEs. AEs were graded as severe, moderate, mild, slight, and none. Pain at the donation site, low back pain, and throat pain were common in BM donors, while low back pain and general malaise were common in PBSC donors. 


\section{Figure 3}

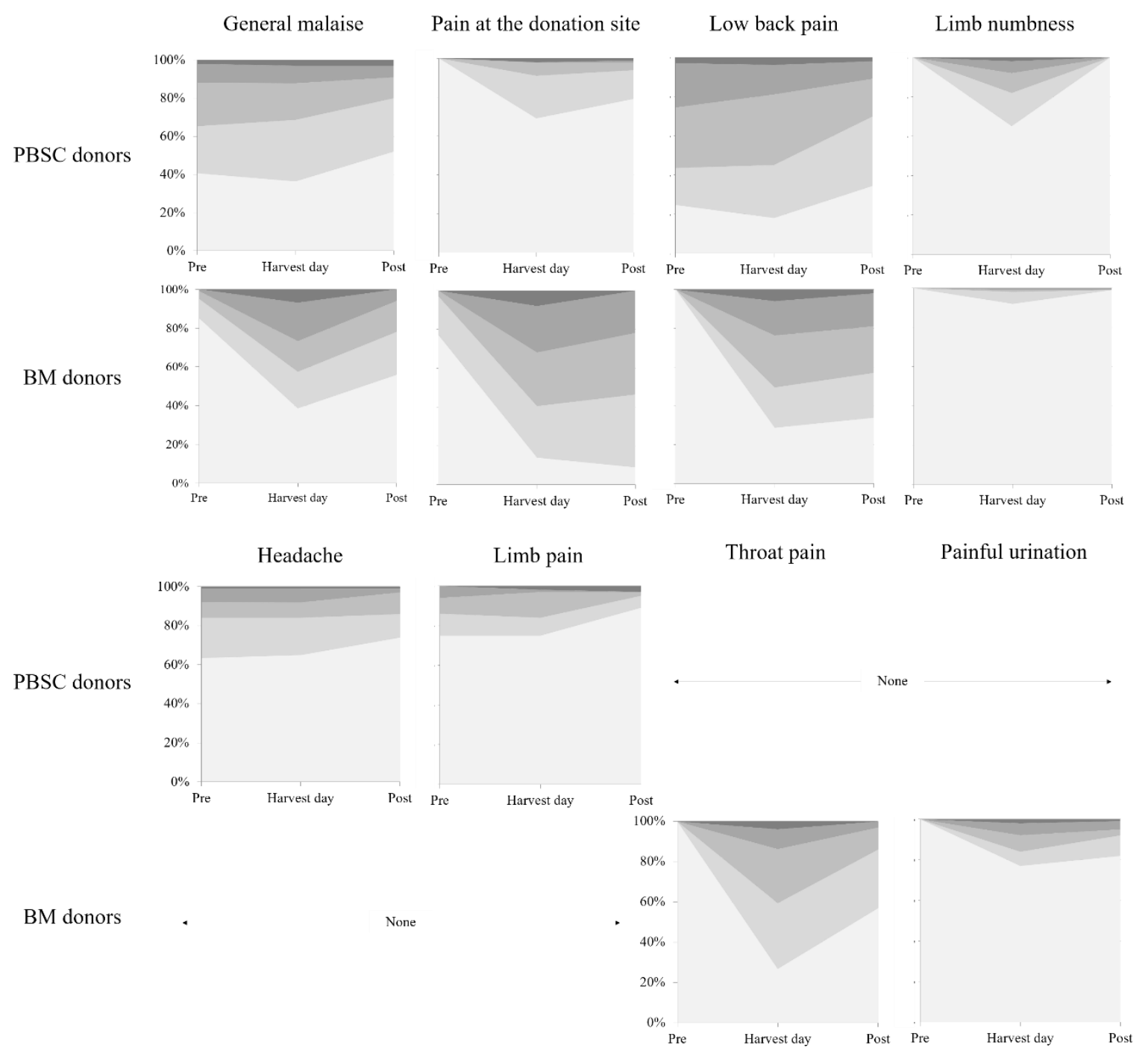

PBSC, peripheral blood stem cells; BM, bone marrow. 
Figure 4 Impact of AEs grade at harvest on the HRQOL after harvest

The development of moderate-to-severe AEs at harvest significantly decrease the total physical score and total mental score 1 week after harvest in both BM and PBSC donors. The impacts lasted until 3 months after harvest only for BM donors. 


\section{Figure 4}

HRQOL at 1 week after harvest

Total physical score

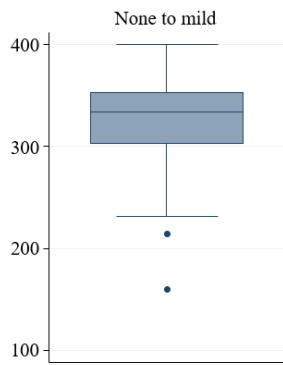

Moderate to severe

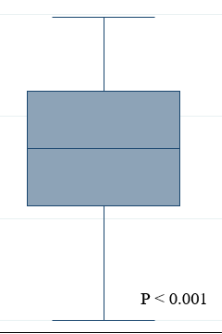

HRQOL at 3 months after harvest
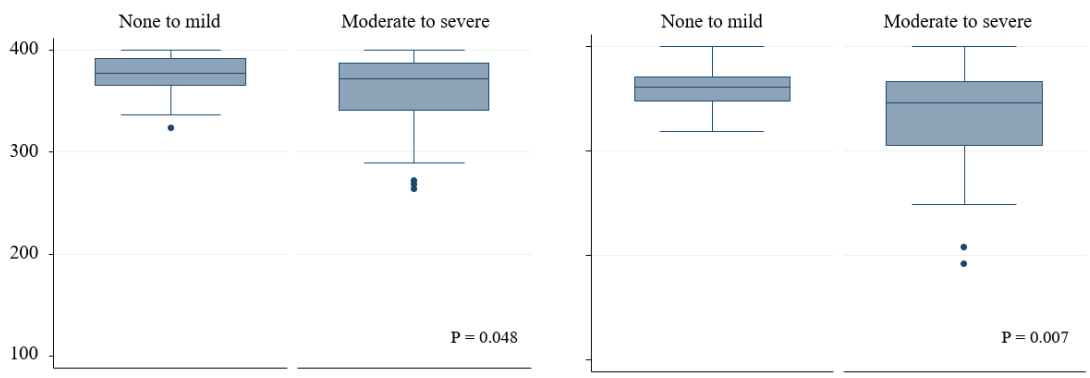

HRQOL at 1 week after harvest

Total physical score

PBSC donors

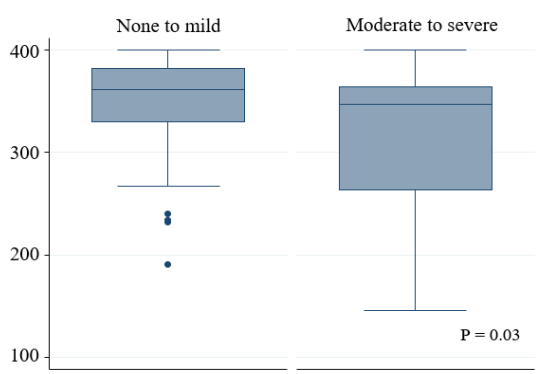

HRQOL at 3 months after harvest

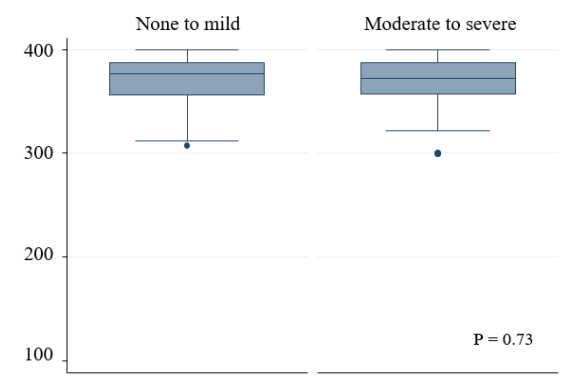

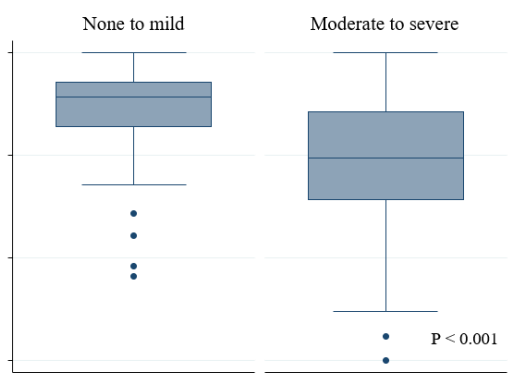

BM donors

Total mental score

(roderate to severe
Total mental score
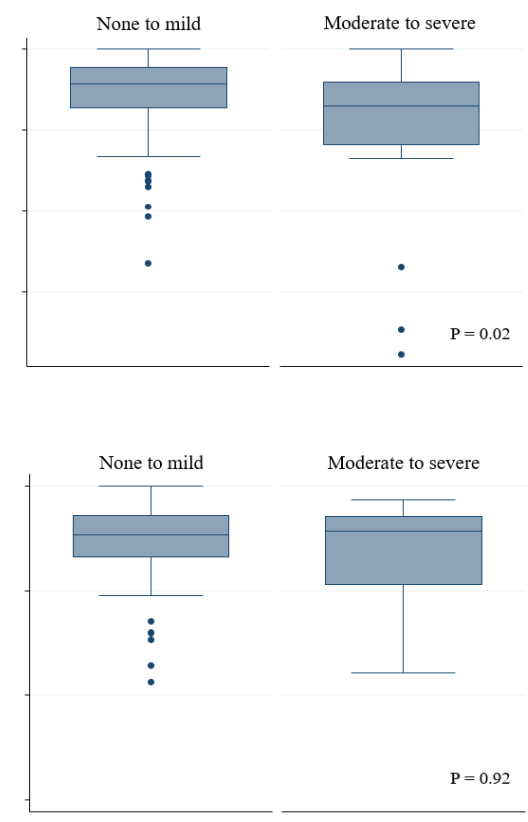